\title{
Perfil epidemiológico do HIV em idosos Brasileiros de 2008 a 2018
}

\author{
Epidemiological profile of HIV in elderly Brazilians from 2008 to 2018 \\ Perfil epidemiológico del VIH en ancianos brasileños de 2008 a 2018
}

Recebido: 07/12/2021 | Revisado: 12/12/2021 | Aceito: 14/12/2021 | Publicado: 22/12/2021

\author{
Gustavo Henrique Andrade \\ ORCID: https://orcid.org/0000-0001-9620-294X \\ Universidade Comunitária da Região de Chapecó, Brasil \\ E-mail: gustavo.andrade@unochapeco.edu.br \\ Luiza Pagnussatt \\ ORCID: https://orcid.org/0000-0002-9141-7375 \\ Universidade Comunitária da Região de Chapecó, Brasil \\ E-mail: luiza.p@unochapeco.edu.br \\ Thalis Matheus Fabiani \\ ORCID: https://orcid.org/0000-0002-3602-0017 \\ Universidade Comunitária da Região de Chapecó, Brasil \\ E-mail: thalis.fabiani@unochapeco.edu.br \\ Vanessa Gheno \\ ORCID: https://orcid.org/0000-0003-2670-0497 \\ Universidade Comunitária da Região de Chapecó, Brasil \\ E-mail: vanessa_g@unochapeco.edu.br \\ Junir Antonio Lutinski \\ ORCID: https://orcid.org/0000-0003-0149-5415 \\ Universidade Comunitária da Região de Chapecó, Brasil \\ E-mail: junir@unochapeco.edu.br
}

\begin{abstract}
Resumo
Introdução: $\mathrm{O}$ aumento da expectativa de vida bem como alterações comportamentais alteraram o perfil epidemiológico do HIV/AIDS no Brasil. Objetivo: identificar a epidemiologia de idosos portadores de HIV/AIDS no Brasil do ano de 2008 até 2018. Metodologia: Trata-se de um estudo epidemiológico realizado por meio de consulta ao DATASUS. Foram consultados os dados do período de 2008 a 2018. Resultados: Neste período foram identificados 21701 novos casos de HIV/AIDS em pacientes com idade $\geq 60$, representando $4,87 \%$ do total, evidenciou-se o crescimento do número de doentes entre os idosos, verificou-se a relação entre o nível de escolaridade e taxa de infecção, o crescimento do número de casos em determinadas regiões e notou-se o processo de heterossexualização da doença. Conclusão: O HIV/AIDS é hoje uma realidade de grandes desafios para gestores e para os profissionais de saúde. Ressalta-se a possibilidade de serem elaboradas e implementadas políticas públicas de prevenção específicas para esta população, bem como o desenvolvimento de uma boa prática profissional capaz de atender o aumento da demanda de idosos que enfrentam esta doença.
\end{abstract}

Palavras-chave: Síndrome da imunodeficiência adquirida; Idosos; Epidemiologia.

\begin{abstract}
Introduction: The increase in life expectancy as well as behavioral changes have changed the epidemiological profile of HIV/AIDS in Brazil. Objective: to identify the epidemiology of elderly people with HIV/AIDS in Brazil from 2008 to 2018. Methodology: This is an epidemiological study carried out through consultation with DATASUS. Data for the period 2008 to 2018 were consulted. Results: In this period, 21701 new cases of HIV/AIDS were identified in patients aged $\geq 60$, representing $4.87 \%$ of the total. elderly, it was verified the relationship between the level of education and the rate of infection, the growth in the number of cases in certain regions and the process of heterosexualization of the disease was noted. Conclusion: HIV/AIDS is today a reality of great challenges for managers and health professionals. We emphasize the possibility of developing and implementing public prevention policies specific to this population, as well as the development of good professional practice capable of meeting the increased demand of elderly people who face this disease.
\end{abstract}

Keywords: Acquired immunodeficiency syndrome; Elderly; Epidemiology.

\section{Resumen}

Introducción: El aumento de la esperanza de vida y los cambios de comportamiento han modificado el perfil epidemiológico del VIH / SIDA en Brasil. Objetivo: identificar la epidemiología de las personas mayores con VIH / SIDA en Brasil de 2008 a 2018. Metodología: Se trata de un estudio epidemiológico realizado a través de la consulta con DATASUS. Se consultaron datos del período 2008 a 2018. Resultados: En este período se identificaron 21701 
nuevos casos de VIH / SIDA en pacientes $\geq 60$ años, lo que representa el 4.87\% del total. y se anotó la tasa de infección, el aumento del número de casos en determinadas regiones y el proceso de heterosexualización de la enfermedad. Conclusión: el VIH / SIDA es hoy una realidad de grandes desafíos para los administradores y profesionales de la salud. Destaca la posibilidad de desarrollar e implementar políticas públicas de prevención específicas para esta población, asî como el desarrollo de una buena práctica profesional capaz de atender la creciente demanda de las personas mayores que enfrentan esta enfermedad.

Palabras clave: Síndrome de inmunodeficiencia adquirida; Anciano; Epidemiología.

\section{Introdução}

A expectativa de vida mundial vem aumentando, com isso a população idosa é cada vez maior. No Brasil no ano de 2019 o número de idosos chegou a 32,9 milhões IBGE (2019). Salienta-se que é considerado idoso o indivíduo com idade maior ou igual a 60 anos em países em desenvolvimento e 65 ou mais em países desenvolvidos, como descrito por Santos (2011).

O HIV/AIDS foi descrito em 1981, nos Estados Unidos. Foi evidenciado em grupos de pacientes jovens, homossexuais masculinos e indivíduos que receberam transfusões sanguíneas, sendo acompanhadas pelo aparecimento de casos em usuários de drogas injetáveis. O HIV pode ser transmitido de várias formas, através da via sexual e sanguínea, perfurocortantes contaminados, transmissão vertical que pode ser durante a gravidez ou ainda de origem materna durante a amamentação, conforme Castillo (2014).

O primeiro caso Brasileiro foi diagnosticado na cidade de São Paulo na década de 1980, como descrito pelo Ministério da Saúde (2021), atingindo rapidamente parte da população. O Brasil é o país que mais concentra infecções por HIV na América Latina, respondendo por $40 \%$ das novas infecções, como visto no programa das Nações Unidas UNAIDS (2016).

Com o aumento na longevidade, tem-se o aumento de novas experiências nesta fase da vida, como a sexualidade facilitada pelo advento de fármacos para impotência, tendo em contraponto os idosos mais vulneráveis a se contaminarem pelo HIV e outras infecções sexualmente transmissíveis (IST), como descrito por Laroque (2011). Quando analisado o tratamento, a terapia antirretroviral tem sido limitada em alguns países, já que 40\% das pessoas que vivem com HIV no mundo não conhecem o estado da sua doença, como descrito pelo programa das Nações Unidas UNAIDS (2016).

No início da epidemia a população idosa não foi atingida, tendo apenas quatro casos notificados no Brasil em pessoas com 60 anos ou mais, porque se considerava que os idosos tinham uma vida sexual inativa, conforme Silva (2013). Entretanto, agora se percebe uma mudança deste padrão, a epidemia atinge a todos, indiferente de orientação sexual e comportamentos. Sendo um dos motivadores os medicamentos para tratamento de disfunção erétil, disponíveis no mercado a partir da década de 1990, proporcionando-lhes vida sexual mais ativa. Também é válido ressaltar que indivíduos mais velhos frequentemente não são percebidos pelos seus médicos como grupo de risco para infecção pelo HIV e consequentemente são menos testados para o HIV quando comparados a adultos mais jovens, como descrito por Grenne (2013). Sabendo-se que apesar do número representativo de novas infecções em adultos mais velhos, poucas campanhas de prevenção têm como alvo específico essa faixa etária, como visto por Autenrieth (2018).

Com isso, tem-se a justificativa da avaliação deste panorama geral entre os idosos no período entre 2008 e 2018 , população que já tem, em grande parte, sua saúde comprometida e precisa de cuidados específicos.

Este trabalho tem como objetivo identificar a epidemiologia dos portadores de HIV no Brasil na população idosa, analisando dados como: número de casos, gênero, local de residência, escolaridade, etnias e a forma de contaminação, bem como as relações com todo o conjunto de portadores.

\section{Metodologia}

Trata-se de um estudo epidemiológico descritivo com abordagem quantitativa, cujos dados foram obtidos por meio de 
consulta de dados disponibilizados pelo Departamento de Informática do Sistema Único de Saúde (DATASUS), no endereço eletrônico (http://www.datasus.gov.br).

É válido enfatizar que no estudo descritivo o pesquisador não interfere sobre os dados coletados, portanto os dados coletados são "observados, registrados, analisados, classificados e interpretados" (Prodanov \&Freitas, 2013, p. 52). Ainda de acordo com Prodanov e Freitas (2013), atrelado ao estudo descritivo se tem a pesquisa quantitativa que utiliza recursos e técnicas estatísticas para quantificar os dados pesquisados e auxiliar na elaboração dos resultados.

A população do estudo foi constituída por pacientes portadores de HIV/AIDS, de todas as faixas etárias, diagnosticadas no período de 2008 a 2018. As variáveis analisadas são: idade, gênero, local de residência, escolaridade, etnias, número de casos e a forma de contaminação. Os critérios de exclusão foram dados incompletos. Tratando-se de um banco de domínio público não foi necessário submeter o projeto ao comitê de Ética em Pesquisa.

\section{Resultados e Discussão}

Na Tabela 1 têm-se os resultados referentes ao número total de portadores de HIV/AIDS de acordo com o ano, demonstrando que no ano de 2008 os idosos representavam 3,82\%, e em 2018 representam 6,32\%.

Tabela 1 - Distribuição total do número de casos de HIV/AIDS diagnosticados por ano, segundo faixa etária, Brasil, 2021

\begin{tabular}{lllll}
\hline Ano Diagnóstico & $<\mathbf{6 0}$ & $\mathbf{2 6 0}$ & Ignorado & Total \\
\hline $\mathbf{2 0 0 8}$ & 39320 & 1.561 & 1 & 40.882 \\
$\mathbf{2 0 0 9}$ & 39144 & 1.647 & 0 & 40.818 \\
$\mathbf{2 0 1 0}$ & 38782 & 1.627 & 0 & 40.409 \\
$\mathbf{2 0 1 1}$ & 40564 & 1.791 & 0 & 42.355 \\
$\mathbf{2 0 1 2}$ & 40206 & 1.879 & 1 & 42.086 \\
$\mathbf{2 0 1 3}$ & 40923 & 2.111 & 0 & 42.934 \\
$\mathbf{2 0 1 4}$ & 39643 & 2.103 & 0 & 41.746 \\
$\mathbf{2 0 1 5}$ & 38364 & 2.142 & 0 & 40.506 \\
$\mathbf{2 0 1 6}$ & 36159 & 2.235 & 0 & 38.924 \\
$\mathbf{2 0 1 7}$ & 35744 & 2.255 & 0 & 37.999 \\
$\mathbf{2 0 1 8}$ & 34811 & 2.350 & 0 & 37.161 \\
\hline TOTAL & 424117 & 21.701 & 2 & 445.82 \\
\hline
\end{tabular}

Fonte: http://www2.aids.gov.br/cgi/deftohtm.exe?tabnet/br.def

Observa-se na Tabela 2 o número de idosos portadores de HIV/AIDS, nota-se uma certa estabilidade em regiões como Sudeste, Centro Oeste, em contraponto há um grande aumento no número de casos nas demais regiões. 
Tabela 2 - Distribuição do número de casos de HIV/ AIDS em pacientes com $\geq 60$ de acordo com a região, Brasil, 2021.

\begin{tabular}{cccccccc}
\hline $\begin{array}{c}\text { Ano } \\
\begin{array}{c}\text { Diagnóstic } \\
\text { o }\end{array}\end{array}$ & $\begin{array}{c}\text { Região } \\
\text { Norte }\end{array}$ & $\begin{array}{r}\text { Região } \\
\text { Nordeste }\end{array}$ & $\begin{array}{c}\text { Região } \\
\text { Sudeste }\end{array}$ & $\begin{array}{c}\text { Região } \\
\text { Sul }\end{array}$ & $\begin{array}{c}\text { Região } \\
\text { Centro- } \\
\text { Oeste }\end{array}$ & $\begin{array}{c}\text { Ignorado/Exteri } \\
\text { or }\end{array}$ & Total \\
\hline $\mathbf{2 0 0 8}$ & 48 & 136 & 488 & 284 & 80 & 525 & 1.561 \\
$\mathbf{2 0 0 9}$ & 58 & 159 & 566 & 315 & 74 & 475 & 1.647 \\
$\mathbf{2 0 1 0}$ & 60 & 172 & 537 & 329 & 71 & 458 & 1.627 \\
$\mathbf{2 0 1 1}$ & 75 & 214 & 576 & 380 & 86 & 460 & 1.791 \\
$\mathbf{2 0 1 2}$ & 70 & 233 & 547 & 416 & 115 & 498 & 1.879 \\
$\mathbf{2 0 1 3}$ & 103 & 285 & 591 & 398 & 119 & 615 & 2.111 \\
$\mathbf{2 0 1 4}$ & 107 & 278 & 533 & 381 & 118 & 686 & 2.103 \\
$\mathbf{2 0 1 5}$ & 96 & 273 & 537 & 398 & 91 & 747 & 2.142 \\
$\mathbf{2 0 1 6}$ & 101 & 285 & 524 & 376 & 89 & 860 & 2.235 \\
$\mathbf{2 0 1 7}$ & 107 & 262 & 502 & 353 & 107 & 924 & 2.255 \\
$\mathbf{2 0 1 8}$ & 104 & 295 & 477 & 354 & 87 & 1.033 & 2.350 \\
\hline TOTAL & 929 & 2.592 & 5.878 & 3.984 & 1.037 & 7.281 & 21.701 \\
\hline
\end{tabular}

Fonte: http://www2.aids.gov.br/cgi/deftohtm.exe?tabnet/br.def.

Na Tabela 3 é discriminada a quantidade de infectados por sexo. Nota-se certa estabilidade na relação dos sexos. Quanto à escolaridade dos idosos portadores na Tabela 4 é demonstrado que quanto menor o nível de escolaridade maior o número de infecções. Já em relação à categoria de exposição hierarquizada existe um número maior de idosos heterossexuais portadores de HIV/AIDS como visto na Tabela 5.

Tabela 3 - Distribuição do número de portadores de HIV/AIDS pelo sexo e ano de diagnóstico em pacientes com $\geq 60$ anos, Brasil, 2021.

\begin{tabular}{llll}
\hline Ano Diagnóstico & Masculino & Feminino & Total \\
\hline 2008 & 943 & 618 & 1.561 \\
2009 & 975 & 672 & 1.647 \\
2010 & 963 & 664 & 1.627 \\
2011 & 1.047 & 744 & 1.791 \\
2012 & 1.141 & 738 & 1.879 \\
2013 & 1.296 & 815 & 2.111 \\
2014 & 1.292 & 811 & 2.103 \\
2015 & 1.314 & 828 & 2.142 \\
2016 & 1.367 & 868 & 2.235 \\
2017 & 1.395 & 860 & 2.255 \\
2018 & 1.491 & 859 & 2.350 \\
\hline TOTAL & 13.224 & 8.477 & 21.701 \\
\hline
\end{tabular}

Fonte: http://www2.aids.gov.br/cgi/deftohtm.exe?tabnet/br.def. 
Tabela 4 - Distribuição conforme orientação sexual e modo de contaminação com o HIV/AIDS em pacientes $\geq 60$ anos, Brasil, 2020 .

\begin{tabular}{|c|c|c|c|}
\hline $\begin{array}{l}\text { Categoria } \\
\text { Exposição } \\
\text { Hierarquizada }\end{array}$ & $\mathrm{N}^{\circ}$ em 2008 & $N^{o}$ em 2018 & $\begin{array}{c}\text { Total de } 2008 \\
\text { até } 2018\end{array}$ \\
\hline Homossexual & 61 & 63 & 793 \\
\hline Bissexual & 35 & 54 & 519 \\
\hline Heterossexual & 702 & 915 & 9.901 \\
\hline $\begin{array}{l}\text { Usuários de } \\
\text { dispositivos } \\
\text { injetáveis }\end{array}$ & 8 & 12 & 120 \\
\hline Hemofílico & 0 & 0 & 2 \\
\hline Transfusão & 3 & 1 & 11 \\
\hline $\begin{array}{l}\text { Acid. Material } \\
\text { Biológico }\end{array}$ & 0 & 0 & 1 \\
\hline $\begin{array}{l}\text { Transmissão } \\
\text { vertical }\end{array}$ & 3 & 7 & 55 \\
\hline Ignorado & 749 & 1298 & 10.299 \\
\hline TOTAL & 1.561 & 2.350 & 21.701 \\
\hline
\end{tabular}

Fonte: http://www2.aids.gov.br/cgi/deftohtm.exe?tabnet/br.def.

Tabela 5 - Distribuição do número de casos conforme escolaridade em pacientes com $\geq 60$ anos, Brasil, 2020.

\begin{tabular}{ll}
\hline Escolaridade & $\mathbf{N}^{\mathbf{o}}$ \\
\hline Analfabeto & 1.093 \\
$\mathbf{1}^{\mathbf{a}}$ a $\mathbf{4}^{\mathbf{a}}$ série incompleta & 2.175 \\
$\mathbf{4}^{\mathbf{a}}$ série completa & 1.262 \\
$\mathbf{5}^{\mathbf{a}}$ a $\mathbf{8}^{\mathbf{a}}$ série incompleta & 2.060 \\
Fundamental completo & 1.231 \\
Médio incompleto & 467 \\
Médio completo & 1.155 \\
Superior incompleto & 138 \\
Superior completo & 715 \\
\hline TOTAL & 10.296 \\
\hline
\end{tabular}

Fonte: http://www2.aids.gov.br/cgi/deftohtm.exe?tabnet/br.def.

Em relação à distribuição de casos conforme faixa etária entre os indivíduos com menos de 60 anos existe um número total de 424.117 sendo 276.600 do sexo masculino e 147.458 feminino, 59 formulários em branco. Entre idosos anos há um total de 21.701, sendo 13.224 do sexo masculino e 8.477 do sexo feminino. Já em relação à distribuição de casos conforme raça cor em idosos há um número total de 21.701, sendo que 38,5\% foram ignorados; brancos 32,5\%; pretos 6\%; amarelos $0,5 \%$; pardos $22.25 \%$; indígenas $0.25 \%$.

Os idosos infectados por HIV/AIDS no Brasil são cada vez um número maior, seja pela política brasileira de acesso universal à terapia antirretroviral (TARV) que possibilitou maior sobrevida aos portadores, ou seja pela falta de utilização de preservativo entre os idosos, como descrito por Beck (2014). Desde 2008, nota-se um aumento no número de casos e na 
porcentagem que eles representam, como evidenciado nesta pesquisa, na qual em 2008 os idosos eram 3,81\% (1561) do total de casos (40882) e em 2018 representavam 6,32\% (2350) do total de casos (37161). Um aumento de 65\% em relação a representatividade dos casos nos portadores com idade superior ou igual a 60 anos e um aumento de 50,54\% no número de casos nestes dez anos.

Um estudo que foi realizado com pacientes portadores de HIV demonstrou que a infecção é diagnosticada de forma tardia nos idosos, sendo realizada após uma longa investigação e como um diagnóstico de exclusão de outras doenças, pois esta doença se manifesta por sinais e sintomas frequentemente confundidos com de outras comorbidades e levando-se em conta que os idosos estão menos propensos a serem testados em comparação a adultos jovens, como observado por Greene (2014). Devese levar em conta que o maior acesso aos medicamentos para distúrbio erétil tem contribuído para desmistificação da hipoatividade sexual entre idosos bem como para o aumento da transmissão de infecções sexualmente transmissíveis (IST), como descrito por Santos (2011). Além disso, as alterações próprias do envelhecimento tornam o sistema imunológico deprimido, tornando o idoso mais suscetível a enfermidades e menos responsivo a elas. E ainda, levando-se em conta que sinais e sintomas inespecíficos como anorexia e astenia, comuns em idosos, dificultam o diagnóstico precoce do HIV/AIDS, como visto por Santos (2011).

Em relação às regiões em que se encontram estes idosos infectados chama a atenção como em dez anos ocorreu o aumento, respectivamente de 116\%, 116\%, 18\%, nas regiões Norte, Nordeste e Sul, na Região Sudeste houve regressão do número de casos e na região centro oeste um aumento pouco sensível de 7 casos. Em contrapartida, o Boletim Epidemiológico (2016) descreve a região sul do Brasil como o local com as maiores taxas de detecção de AIDS, destacando o estado do Rio Grande do Sul, o que demonstra como as diferenças entre a disponibilidade de serviços de saúde em cada região, preparo das pessoas que realizaram a notificação e a presença de políticas públicas pode ser diferente nas regiões, evidenciando que estas questões merecem maior discussão e estudo.

Quando analisados dados em relação ao sexo dos portadores de HIV/AIDS com idade igual ou superior a 60 anos no período de 2008 a 2018, nota-se 38\% de aumento entre os casos das mulheres e 58\% em relação aos homens, em relação ao total de casos em 2008 as mulheres representavam 39\%, já em 2018 representam 36\%. Isso é explicado pela falta de conscientização entre os homens acerca da importância em acessar os serviços de prevenção e assistência. Além disso, o homem tende a considerar-se imune à doença, rejeitando a possibilidade de adoecer, com isso fica suscetível ao adoecimento, como visto no Boletim Epidemiológico HIV/Aids (2015) e Amaral (2018).

Quando são discriminados os dados dos sexos de portadores de HIV/AIDS, com idade igual ou superior a 60 anos e idade inferior a 60 anos, tem-se que $34.8 \%$ dos portadores são mulheres já em idade igual ou superior a 60 anos representam $40 \%$, nota-se pequena elevação no percentual representativo de mulheres idosas portadoras.

Ao cruzar os dados do nível de escolaridade fica clara a relação entre o acesso à informação e os níveis de contaminação da doença, pois $80 \%$ dos portadores possuem nível de ensino inferior ao médio incompleto. Indo em acordo com dados que caracterizam a epidemiologia do HIV/AIDS com baixo nível de escolaridade, como descrito por Gomes (2017). É de conhecimento que no Brasil a prevalência de HIV/AIDS está intrinsecamente envolvida com a baixa intrusão e vulnerabilidade econômica. Em contraponto, quanto maior a escolaridade maior o estímulo e acesso a conhecimentos sobre riscos à saúde e transmissibilidade de doenças, conforme a descrição de Gomes (2017). Um estudo realizado em Botswana, na África, entre os anos de 2004 e 2008 sugeriu que aumentar a progressão no ensino médio pode ser uma medida de prevenção do HIV, também constatou que a cada ano adicional de ensino médio causado pela mudança de política levou a uma redução absoluta no risco cumulativo de infecção pelo HIV, principalmente entre as mulheres, de 8,1 pontos percentuais em relação a uma prevalência inicial de 25,5\% no período pré-escolar, como observado por De Neve (2015). 
Ao analisar os dados referentes a raça/cor dos idosos portadores nota-se um número maior de pacientes da cor branca $52 \%$, seguindo pela parda $35 \%$, excluindo os casos com prontuários incompletos que somam $38 \%$, o que implica em uma crítica à forma de registro e alimentação dos bancos de dados, devido ao grande número de prontuários incompletos. Quando comparado a um estudo Brasileiro dos anos de 2005 até 2012 demonstra semelhança, neste estudo a cor branca representava 46,9\% seguindo pela parda com 40,8\%, como visto por Dartora (2017). Já em relação a um estudo realizado nos Estados Unidos entre 2006 e 2009 apresentou certa discrepância, pois foi encontrado um número maior entre negros/afro-americanos e hispânicos do que brancos com taxas de 7,1 e 3,0 em 2006; 7,4 e 2,8 em 2007; 8,4 e 3,0 em 2008; e 7,7 e 2,9 em 2009, respectivamente, como observado por Prejan (2011)'. Diferença explicada pela população brasileira ser majoritariamente de brancos e pardos. Sendo 45,5\% se declaram brancos e 45\% pardos de acordo com a pesquisa Nacional de Amostra por Domicílio do IBGE (2021).

Com relação à categoria de exposição entre os idosos notamos uma heterossexualização da doença com um crescimento de $30 \%$ em 10 anos, enquanto entre os homossexuais foi de 3,27\%. Quando analisado o total de casos entre 2008 e 2018 temos $86 \%$ de heterossexuais e 6,95\% de homossexuais, seguidos pelos bissexuais com 4,55\%, seguindo o processo de heterossexualização da epidemia, como já observado por Knauth (2020). Os homens homossexuais, bissexuais e outros que fazem sexo com homens continuam a ter elevadas taxas de infecção pelo HIV em países de baixa, média e alta renda, como descrito por Beyrer (2016). Chama atenção a categoria de transmissão vertical descrita pelo DATASUS já que por se tratar de idosos é imprudente dizer que ocorreu a transmissão mãe para feto, sendo que o primeiro caso foi descrito em 1980, posterior ao nascimento da população descrita.

O DATASUS tem por objetivo a disponibilização dos dados de acordo com a Política Nacional de Informação e Informática em Saúde que tem como um de seus princípios o acesso à informação em saúde como um direito a todo o indivíduo. As limitações do estudo encontram-se no uso de dados secundários, permitindo possíveis subnotificações, erros de digitação e de registro. Em contraponto acredita-se que por se tratarem de dados nacionais e oficiais de preenchimento obrigatório em todos os serviços de saúde, os resultados permitiram o alcance dos objetivos propostos.

\section{Conclusão}

No presente estudo foram identificados os dados epidemiológicos do HIV. Descreveu-se um aumento de 50,54\% no número de casos na população idosa. Notou-se que as regiões Norte, Nordeste e Sul tiveram um aumento no número de casos, enquanto no Sudeste houve regressão e no Centro Oeste ficou próximo da estabilidade. A relação entre os sexos permaneceu próxima da estabilidade com discreta masculinização do HIV nos idosos. Observou-se que quanto menor o nível de escolaridade maiores são as taxas de contaminação. Os dados referentes a raça/cor revelaram que os portadores são $52 \%$ brancos, seguidos dos pardos com 35\%. Já em relação à categoria de exposição notou-se um aumento na porcentagem que representam os heterossexuais. Diante do descrito, aponta-se a necessidade da implantação de políticas públicas abrangentes e funcionais para a população idosa, discutindo as formas de transmissão e a gravidade do HIV.

Sugere-se para trabalhos futuros a análise de como intervenções focadas na população idosa podem interferir nas taxas de infecção do HIV. É de suma importância a conscientização quanto ao uso do preservativo, bem como de outros métodos. Além disso, é fundamental que os profissionais de saúde solicitem o exame para diagnóstico da doença e que haja um preenchimento adequado das fichas de notificação, uma vez que são ferramentas imprescindíveis para o estudo epidemiológico nacional.

\section{Referências}

Autenrieth, C. S., Beck, E. J., Stelzle, D., Mallouris, C., Mahy, M., \& Ghys, P. (2018). Global and regional trends of people living with HIV aged 50 and over: Estimates and projections for 2000-2020. PloS one, 13(11), e0207005. 
Beck, C. (2014). Análise de sobrevida e perfil epidemiológico de casos de AIDS em Porto Alegre / RS: limitações e potencialidades da vigilância epidemiológica. Lume.ufrgs.br, 7531 (415). http://hdl.handle.net/10183/131161

Beyrer, C., Baral, S. D., Collins, C., Richardson, E. T., Sullivan, P. S., Sanchez, J., ... \& Mayer, K. H. (2016). The global response to HIV in men who have sex with men. The Lancet, 388(10040), 198-206.

Ministério da Saúde (2015). Departamento de IST, HIV/AIDSe Hepatites Virais: Boletim Epidemiológico AIDSe DST.

Castillo, E. R. (2014). Modeling the Dynamics of a Group of Positive HIV Individuals with Type-2 Fuzzy Parameter.. Dissertação (Mestrado em Ciências Exatas e da Terra) - Universidade Federal de Uberlândia, Uberlândia, 96 f. https://doi.org/10.14393/ufu.di.2014.273

Da Silva Amaral, R., de Carvalho, S. T. R. F., Silva, F. D. M. A. M., \& da Silva Dias, R. (2017). Soropositividade para hiv/aids e características sociocomportamentais em adolescentes e adultos jovens/hiv/aids and sociocomportamental characteristics of young adolescents and adults. Revista de Pesquisa em Saúde, 18(2).

Dartora, W. J., nflor, É. P., \& da Silveira, L. R. P. (2017). Prevalência do HIV no Brasil 2005-2015: dados do Sistema Único de Saúde. Revista Cuidarte, 8(3), $1919-1928$

Departamento de Informática do Sistema Único de Saúde -DATASUS. (2019). https://datasus.saude.gov.br/

De Neve, J. W., Fink, G., Subramanian, S. V., Moyo, S., \& Bor, J. (2015). Length of secondary schooling and risk of HIV infection in Botswana: evidence from a natural experiment. The Lancet Global Health, 3(8), e470-e477.

Gomes, R. R. D. F. M., Ceccato, M. D. G. B., Kerr, L. R. F. S., \& Guimarães, M. D. C. (2017). Fatores associados ao baixo conhecimento sobre HIV/AIDS entre homens que fazem sexo com homens no Brasil. Cadernos de Saúde Pública, 33, e00125515.

Greene, M., Justice, A. C., Lampiris, H. W., \& Valcour, V. (2013). Management of human immunodeficiency virus infection in advanced age. Jama, 309(13), 1397-1405.

Departamento de Informática Instituto Brasileiro de Geografia e Estatística. (2020). Portal do Instituto Brasileiro de Geografia e Estatística. Recuperado em 2 abril, 2020, de http://www.ibge.gov.br

Knauth, D. R., Hentges, B., Macedo, J. L. D., Pilecco, F. B., Teixeira, L. B., \& Leal, A. F. (2020). O diagnóstico do HIV/aids em homens heterossex uais: a surpresa permanece mesmo após mais de 30 anos de epidemia. Cadernos de Saúde Pública, 36, e00170118.

Laroque, M. F., Affeldt,. B., Cardoso, D. H., Souza, G. L. D., Santana, M. D. G., \& Lange, C. (2011). Sexualidade do idoso: comportamento para a prevenção de DST/AIDS. Revista Gaúcha de Enfermagem, 32, 774-780.

Ministério da Saúde, Brasil. Secretaria de Vigilância em Saúde. Departamento Nacional de DST, Aids e Hepatites Virais. Boletim Epidemiológico Aids/DST. Brasília: Ministério da Saúde. 2016

Prejean, J., Song, R., Hernandez, A., Ziebell, R., Green, T., Walker, F., ... \& HIV Incidence Surveillance Group. (2011). Estimated HIV incidence in the United States, 2006-2009. PloS one, 6(8), e17502.

Prodanov, C. C., Freitas, E. C. (2013). Metodologia do trabalho científico: métodos e técnicas da pesquisa e do trabalho acadêmico (2ª ed.). Novo Hamburgo: Feevale.

Santos, A. F. D. M., \& Assis, M. D. (2011). Vulnerabilidade das idosas ao HIV/AIDS: despertar das políticas públicas e profissionais de saúde no contexto da atenção integral: revisão de literatura. Revista Brasileira de Geriatria e Gerontologia, 14, 147-157.

Augusto, R., Da Silva, R., Hiago, F., Duarte, S., Raquel, A., Nelson, C., Rebberty, J., \& Holanda, R. (6039). A epidemia da aids no brasil: análise do perfil atual aids epidemic in brazil: analysis of current profile la epidemia del sida en brasil: análisis del perfil actual artigo informativo. Rev Enferm UFPE on Line, 7(19818963). https://doi.org/10.5205/reuol.4377-36619-1-ED.0710201327

UNAIDS. Prevention gap report 2016. Geneva: AIDS by the numbers. 2016.

UNAIDS. Report on the global AIDS epidemic 2016. Geneva: AIDS by the numbers. 2016. 